\title{
Removal of Debris and Smear Layer in Curved Root Canals Using Self-Adjusting File with Different Operation Times A Scanning Electron Microscope Study
}

\author{
Senem YİĞìt ÖZER ${ }^{1}$, Özkan ADIGÜZEL ${ }^{1}$, Sadullah KAYA ${ }^{1}$ \\ ${ }^{1}$ Assistant Professor, Dicle University, Faculty of Dentistry, Department of Operative Dentistry and Endodontics, \\ Diyarbakır, TURKEY
}

\begin{abstract}
Aim: Debridement during root canal treatment is mandatory and it is provided by means of chemomechanical instrumentation and irrigation methods. This article analysis the debridement capacity of a novel system, SAF and its special irrigation device when used with different operation times in curved root canals.

Methodology: 30 mesiobuccal root canals of maxillary molars were instrumented using SAF. Teeth were divided into three groups. In Group 1, 10 new SAF files were used for operation for 4 minutes. In Group 2, the 4-min previously used SAF files were operated in the same manner. In Group 3, the 8-min previously used SAF files were operated. During SAF operation $2.6 \% \mathrm{NaOCl}$ and $17 \%$ EDTA were used alternately in all groups. Debris and smear layer removal were evaluated for the apical thirds under scanning electron microscope.
\end{abstract}

\section{Key Words}

Apical third of root canal, curved root canal, debridement, self-adjusting file, smear layer

\author{
Correspondence: \\ Senem YİĞİT ÖZER \\ Dicle University, \\ Faculty of Dentistry, \\ Department of Operative Dentistry \\ and Endodontics, \\ 21280, Diyarbakir, TURKEY. \\ e-mail: senemygt@hotmail.com
}

Results: Non-used, 4-min preused, and 8-min preused SAF efficiently removed debris and smear layer in the apical thirds. There were no significant difference among the groups in terms of debridement.

Conclusions: When SAF is operated in curved root canals with continous flow of irrigation it results in debris and smear-free canal walls in the critical apical thirds within 12 minutes.

(Int Dent Res 2011;1:1-6)

\section{Introduction}

Root canal instrumentation produces smear layer. This amorphous structure is composed of dentin particles, necrotic debris, and odontoblastic processes that occlude the orifices of dentinal tubules (1). Smear layer is reported to prevent the penetration of irrigation solutions, medications, and filling materials into dentinal tubules and many researchers believe that it is detrimental (2). The literature reports generally show that regardless of the instrumentation and irrigation techniques, the effectiveness of irrigating solutions remains limited in the apical one third of a prepared canal. This is particularly true for curved root canals (3). Therefore, the improvement of irrigating protocols is essential during root canal treatment in order to achieve better cleaning efficiency especially in the very complex apical area.

Rotary nickel-titanium files are successful to clean and shape the straight and narrow canals, and completion of the file sequence may result in a clean canal with no tissue debris and with removal of all or most of the inner layer of the heavily contaminated dentin (4). Recently micro-computed tomographic studies by Peters et al (5) have extended the 
understanding of the limitations of rotary file systems reporting that inadequate preparation often occurs in curved root canals. In upper molars treated with a conventional rotary system, $49 \%$ of the canal walls were reported to be untouched, even in the larger palatal canals (6). To overcome this handicap, these common nickel-titanium file designs are being modified for a higher percent of the root canal surface to be prepared by the shaping procedure (7).

Self-Adjusting file (SAF) is a novel system among the nickel-titanium files operating in a different manner. It adapts itself longitudinally to a curved canal, as most rotary nickel-titanium files do, but differently adapts itself to the cross-section of the canal (7). It is a hollow file designed as a compressible, thin-walled, pointed cylinder, composed of a thin derivate of nickel-titanium lattice with high torsional and fatigue resistance. The lattice surface is slightly abrasive and it allows removing dentin with a back-and-forth grinding motion (8). This reciprocating file system is used with a specially designed irrigation device providing continuous flow of the irrigant.

During the operating procedure, SAF is inserted into the canal while vibrating and is lightly pushed in until it reaches the predetermined working length. It is then operated with in-and-out manual motion and with continuous irrigation using two cycles of 2 minutes each for a total of 4 minutes per canal. This procedure is reported to remove a uniform dentin layer 60 - to $75-\mathrm{mm}$ thick from the canal circumference (8).

Every available rotary file systems are reported to generate a smear layer leaving debris in the root canal (9) however a recent study by Metzger et al. (10), who used the SAF system with a 4-min application of $3 \% \mathrm{NaOCl}$ and $17 \%$ EDTA reported clean and mostly smear layer-free dentinal surface in all parts of the root canal. However the evaluative tests showed the efficacy of the SAF file declined with time. A file that was preused for 30 minutes was found to be $40 \%$ less effective than a new file (8). Nevertheless, when used for 12 minutes, according to the manufacturer's instructions, the SAF efficacy was not reported to substantially reduce. However the ability to remove dentin is claimed to decrease if the file was reused (8).

Thus the aim of this present study was to evaluate the debridement capacity of SAF when used in curved root canals in an operation time as advised by the manufacturer. The null hypothesis is when SAF is used within 12 minutes it removes smear layer and debris efficiently in the apical thirds of curved root canals.

\section{Materials and Methods}

\section{Selection of Teeth}

The study sample consisted of 30 maxillary molars with fully formed apices that had been extracted for periodontal and/or prosthetic reasons. The teeth were stored in $10 \%$ buffered formalin until they were used. The mesiobuccal (MB) root canals of maxillary molars were instrumented using SAF. Curvature of the MB canals was measured according to the protocoldescribed previously by Estrela et al. (11). The 30 canals showed curvatures ranging from 32 to $45^{\circ}$. This sample was equally divided into 3 groups of 10 teeth for instrumentation with SAF. The working length of each canal was determined by subtracting $1 \mathrm{~mm}$ from the observed length of protrusion of the number 10 file through the apical foramen.

\section{Root Canal Instrumentation and Irrigation with the SAF}

After endodontic accesss cavity, the root canal was negotiated using a size $10 \mathrm{~K}$-file. The working lengths were set $1 \mathrm{~mm}$ shorter than the apical foramen. A glide path was established by manual instrumentation up to a size $20 \mathrm{~K}$-file. The $10 \mathrm{SAF}$ files were operated using an in-and-out vibrating handpiece as described by Metzger et al. (6) with 5000 vibrations/min and a 0.4-mm amplitude, with the irrigation device (VATEA; ReDent-Nova) that provided flow of the irrigation solution at a flow rate of $5 \mathrm{~mL} / \mathrm{min}$ until it reached the predetermined working length for 4 minutes. The irrigation solution flowed into the file and freely escaped into the root canal through the lattice wall to backflow coronally without positive pressure. Because a flow rate of 5 $\mathrm{mL} / \mathrm{min}$ was chosen, $15 \mathrm{~mL}$ of $\mathrm{NaOCl}(2.6 \%)$ and 5 $\mathrm{mL}$ of EDTA (17\%) were used. $\mathrm{NaOCl}$ was used as the initial irrigant during the first $3 \mathrm{~min}$ of the operation, followed by 1 min of irrigation with EDTA. A final flush with $5 \mathrm{~mL} \mathrm{NaOCl}$ was used to remove the EDTA, and distilled water was used in the last step. The canals were dried using paper points.

In Group 1, 10 new SAF files were used for operation for 4 minutes. In Group 2, the 4-min previously used SAF files were operated in the same manner. In Group 3, the 8-min previously used SAF files were operated.

\section{SEM Evaluation}

Two longitudinal grooves were prepared on the buccal and lingual surfaces of each root using a diamond disc, avoiding penetration into the canal. The roots were then split into 2 halves with a chisel and coded. The coded specimens were mounted on metallic stubs, gold sputtered, and examined 
independently by two observers using SEM (Leo Stereoscan S440, Leica, Wetzlar, Germany). After general evaluation of the canal wall, 2 SEM photomicrographs were taken at magnifications of $2000 \times$ at the apical (2 mm to apex) thirds of each specimen for the smear layer and 200x for debris. Cleanliness was evaluated using a 5-point scoring system introduced by Hülsmann et al (12). (1) score 1: no smear layer, and all dentinal tubules were open; (2) score 2: a small amount of smear layer, and some dentinal tubules were open; (3) score 3: homogenous smear layer covering the root canal wall, and only a few dentinal tubules open; (4) score 4: complete root canal wall covered by a homogeneous smear layer, and no open dentinal tubules were observed; and (5) score 5: heavy, homogeneous smear layer covering the complete root canal wall.

The presence of debris was evaluated from images at 200X magnification using a scale of 5 scores (12) as follows: (1) score 1: clean root canal wall and only a few small debris particles, (2) score 2: a few small agglomerations of debris, (3) score 3: many agglomerations of debris covering less than $50 \%$ of the root canal wall, (4) score 4 : more than $50 \%$ of the root canal walls were covered with debris, and (5) score 5: complete or nearly complete root canal wall coverage with debris.

All results were then grouped into "clean canal wall" that included scores 1 and 2 or "smear layer and debris present" that included scores of 3,4, and 5.

Two examiners independently scored each of these images, which were coded and randomly mixed so that the examiners were blinded to the area from which a given sample originated.

When two examiners independently agreed on a score, it was recorded. When disagreement occurred, both two discussed the sample and its scoring, and an agreed score was reached.

\section{Statistical Analysis}

Statistical analysis was performed by using nonparametric analysis of variance. Results were regarded as significant if $p<.05$. Multiple comparisons were adjusted by using the Bonferroni correction.

\section{Results}

\section{Debris}

Root canal preparation using the SAF for three groups rendered all root canals clean of debris. Debris evaluation of the root canal dentinal surfaces usually resulted in debris scores of 1 or 2, representing a clean root canal surface (Figure 1). In the new SAF group $90 \%$ of the apical thirds of the roots were free of debris, this ratio was $87 \%$ in the 8-min used group. Finally 12 -min group was $74 \%$. Statistically there was no difference between the experimental groups $(P>.05)$.

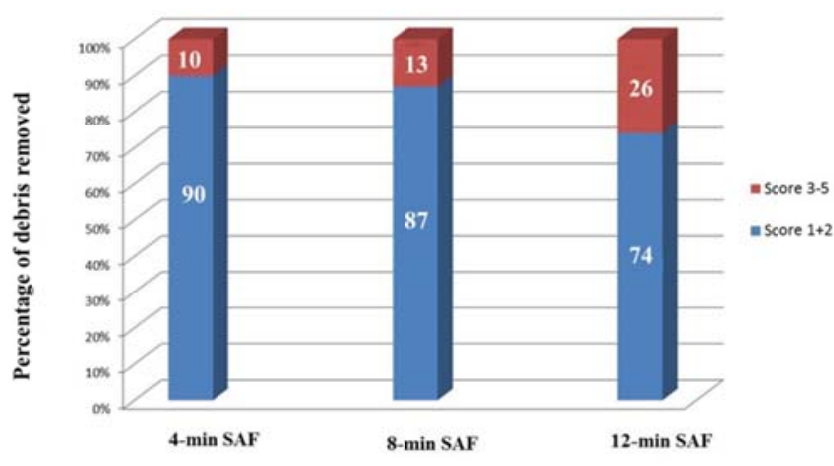

Figure 1. Distribution of debris scores at apical levels. Data were dichotomized for graphic illustration: scores 1-2 (clean canal wall) versus 3-5 (debris present).

\section{Smear Layer}

Operating SAF with 4, 8, and 12-min applications resulted in a root canal surface clean of smear layer (Fig. 2). In the apical thirds, 15 out of 30 were scored as either 1 or 2, respectively, representing a clean dentin surface. 4-min group represented 64\%, 8-min group represented 61\%, and 12 -min group represented $57 \%$ in smear layer scores of 1 or 2, respectively. Different operation time of SAF within 12 minutes removed smear layer almost equally in the apical thirds. No differences between groups were detected statistically $(P>.05)$.

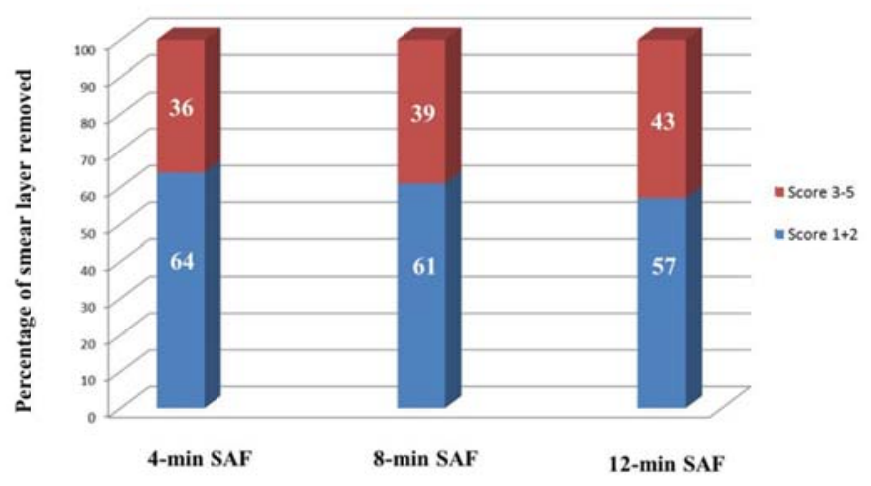

Figure 2. Distribution of smear layer scores at apical levels. Data were dichotomized for graphic illustration: scores 1-2 (clean canal wall) versus 3-5 (smear layer present). 


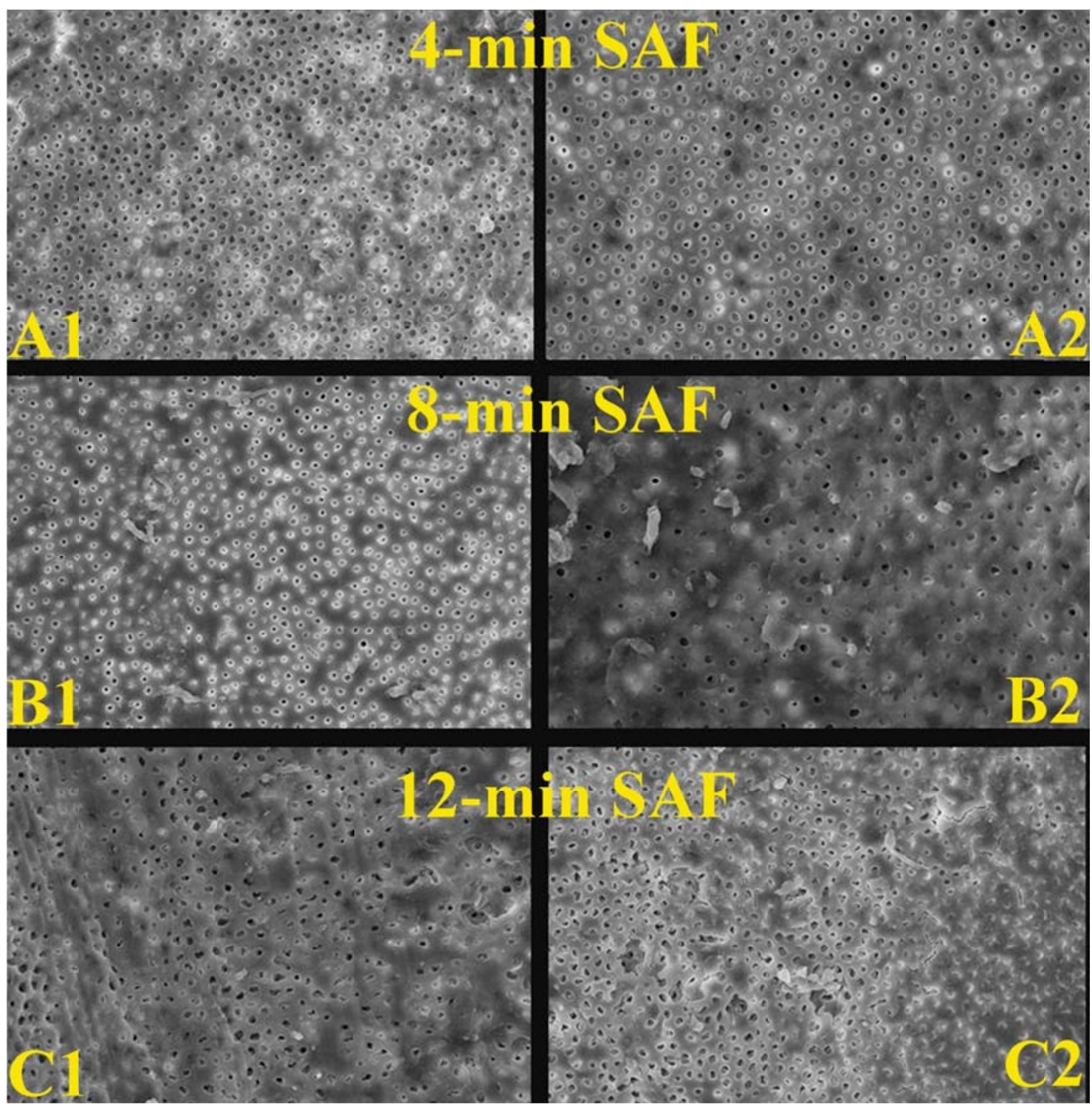

Figure 3. The smear layer in the apical third of curved root canal (A1-2) After 4-min operation of new SAF + dual-irrigation regime the root canal surface is free of debris and smear layer leaving the dentinal tubules open; (B1-2) the 4-min preused SAF were used with the dual-irrigation regime. Most of the dentinal tubules were open with a score 1-2; (C1-2) the 8-min preused SAF + dual-irrigation regime representing score 2-3.

\section{Discussion}

Debridement of the root canal system is a major concern for endodontic success and irrigation is an important part of root canal debridement especially in curved root canals (13). Unfortunately, many studies have reported that currently used methods of root canal preparation and irrigation do not effectively debride the entire root canal system (9). Ideally, root canal irrigants should flush out debris, dissolve organic tissue, destroy microbial byproducts, and remove the smear layer. Past studies have shown that current canal preparation and irrigation methods may be effective at cleaning the coronal portions of root canals but much less effective in the apical portions of canals $(14,15)$. Although several studies indicate that achieving this goal in the apical third of the root canal may be difficult if not impossible, the use of the SAF in combination with a dual-irrigation regime of $\mathrm{NaOCl}$ and EDTA is reported to result in clean dentin surfaces in the apical portion of most root canals (10). Recently the study results of Metzger et al. (10) represented $65 \%$ root canal wall free of smear layer for the apical thirds of the root canal. Our results are in line with this previous study. In the 4min used SAF smear layer was removed $64 \%$, 8min used SAF $61 \%$, and 12-min used SAF $57 \%$, respectively.

Sodium hypochlorite has the ability to dissolve organic debris, and destroy microbial byproducts 
(16). EDTA is a chelating agent used to remove the smear layer (17). This dual combination of irrigants has been shown to be effective in debriding and disinfecting root canals as well as other irrigants (18-21). Studies have shown an increased efficacy of canal debridement with increased apical size preparations and increased taper of instruments $(14,22)$. As reported recently by Peters et al $(5)$ the resulting apical size is usually at least equivalent to a \#40 file when SAF is used during preparation in 5minutes. Several studies confirmed that larger apical preparation reduces the bacterial count $(23,24)$ and enhances the effectiveness of irrigation (25). Probably apical preparation performed using SAF and vibrating motion of the file's delicate mesh within the fluid that is continuously replaced had synergist effect for debridement and resulted with clean root canals almost free of smear layer on the critical apical region.

In addition, the role of chlorine should not be overlooked. It is known that chlorine is responsible for the dissolution of organic tissue and the antimicrobial effect of $\mathrm{NaOCl}$ (26). However, chlorine is consumed rapidly during the first phase of tissue dissolution, probably within 2 min $(26,27)$. Therefore regular replenishment and large volumes of $\mathrm{NaOCl}$ are required for successful debridement. During SAF operation with continous irrigation, one should consider that $\mathrm{NaOCl}$ is refreshed every second making it possible for sufficient free chlorine to be present in the root canal to dissolve the organic component of dentine debris. It could be possible that $\mathrm{NaOCl}$ contains enough free chlorine to dissolve the organic component of the dentine debris and despite the increased operation time with the used files $\mathrm{NaOCl}$ had enough flushing effect on debridement especially at the apical portions of the curved root canals. $\mathrm{NaOCl}$ entering the file through a free-rotating hub is continuously replaced during the procedure, thus providing a fresh and totally active irrigation solution. Because positive pressure is absent throughout the root canal system, the solution can easily escape through openings in the lattice of the file (8). The success in removing the smear layer in the apical third, may be due to the vibrating motion of the file's delicate mesh within the fluid that is continuously replaced.

\section{Conclusions}

When SAF is operated in curved root canals with continous flow of irrigation it results in debris and smear-free canal walls in the critical apical thirds within 12 minutes. Thus our null hypothesis is accepted.

\section{References}

1. McComb D, Smith DC. A preliminary scanning electron microscopic study of root canals after endodontic procedures. J Endod 1975;1:238-42.

2. Karagöz-Küçükay I, Bayirli G. An apical leakage study in the presence and absence of the smear layer. Int Endod J 1994;27;87-93.

3. Sedgley CM, Nagel AC, Hall D, et al. Influence of irrigant needle depth in removing bioluminescent bacteria inoculated into instrumented root canals using real-time imaging in vitro. Int Endod J 2005;38:97-104.

4. Wu M-K, van der Sluis LWM, Wesselink PR. The capacity of two hand instrumentation techniques to remove the inner layer of dentin in oval canals. Int Endod J 2003; 36:218-24.

5. Peters OA, Boessler C, Paque' F. Root canal preparation with a novel nickel-titanium instrument evaluated with micro-computed tomography: canal surface preparation over time J Endod 2010;36:1068-72.

6. Peters $\mathrm{OA}$, Peters $\mathrm{CI}$, Schönenberger $\mathrm{K}$, et al. ProTaper rotary root canal preparation: effects of root canal anatomy on final shape analyzed by micro CT. Int Endod J 2003;36:86-92.

7. Metzger Z, Teperovich E, Zary R, et al. Respecting the root canal: a new concept of a Self Adjusting File (SAF). J Endod 2010;36:67990.

8. Hof R, Perevalov V, Eltanani M, Zary R, Metzger Z. The Self Adjusting File (SAF), Part 2: mechanical analysis. J Endod 2010;36:691-96.

9. Torabinejad M, Handisides R, Khamedi AA, et al. Clinical implications of smear layer in endodontics: a review. Oral Surg Oral Med Oral Path Oral Radiol Endod 2002;94: 658-66.

10. Metzger Z, Teperovich E, Cohen R, et al. The Self Adjusting File (SAF). Part 3: Removal of debris and smear layer. A scanning electron microscope study. J Endod 2010;36;697-702.

11. Estrela C, Bueno MR, Sousa-Neto MD, Pécora JD. Method for determination of root curvature radius using cone-beam computed tomography images. Braz Dent J 2008;2:114-8.

12. Hülsmann $M$, Ruümmelin $C$, Schäfers $F$. Root canal cleanliness after preparation with different endodontic handpieces and hand instruments: a comparative SEM investigation. J Endod 1997;23:301-6.

13. Bystrom A, Sundqvist G. Bacteriologic evaluation of the efficacy of mechanical root canal instrumentation in endodontic therapy. Scand J Dent Res 1981;89:321-8.

14. Usman N, Baumgartner JC, Marshall JG. Influence of instrument size on root canal debridement. J Endod 2004;30:110 -2.

15. Walters MJ, Baumgartner JC, Marshall JG. Efficacy of irrigation with rotary instrumentation. J Endod 2002;28:837-9.

16. Hand RE, Smith ML, Harrison JW. Analysis of the effect of dilution on the necrotic tissue dissolution property of sodium hypochlorite. J Endod 1978;4:60-4. 
17. Baumgartner JC, Mader C. A scanning electron microscopic evaluation of four root canal irrigation regimens. J Endod 1987;13:147-52.

18. Bystrom A, Sundqvist G. The antibacterial action of sodium hypochlorite and EDTA in 60 cases of endodontic therapy. Int Endod J 1985;1:35- 40.

19. Baumgartner JC, Mader C. A scanning electron microscopic evaluation of four root canal irrigation regimens. J Endod 1987;13:147-52.

20. Johal S, Baumgartner JC, Marshall JG. Comparison of the antimicrobial effect of $1.3 \%$ $\mathrm{NaOCl} / \mathrm{MTAD}$ with $5.25 \% \mathrm{NaOCl} / 15 \%$ EDTA for root canal irrigation. J Endod 2007; 33:48 -51.

21. Kho $P$, Baumgartner JC. A comparison of the antimicrobial efficacy of $\mathrm{NaOCI} / \mathrm{Biopure} \mathrm{MTAD}$ versus $\mathrm{NaOCI} / \mathrm{EDTA}$ against Enterococcus faecalis. J Endod 2006;32:652-5.

22. Wu MK, Wesselink PR. Efficacy of three techniques in cleaning the apical portion ofcurved root canals. Oral Surg Oral Med Oral Pathol Oral Radiol Endod 1995;79:492- 6.

23. Card SJ, Sigurdsson A, Orstavik D, Trope M. The effectiveness of increased apical enlargement in reducing intracanal bacteria. J Endod 2002; 28:779-83.

24. Rollison S, Barnett F, Stevens RH. Efficacy of bacterial removal from instrumented root canals in vitro related to instrumentation technique and size. Oral Surg Oral Med Oral Pathol Oral Radiol Endod 2002;94:366-71.

25. Chow TW. Mechanical effectiveness of root canal irrigation. J Endod 1983;9:475-9.

26. Moorer WR, Wesselink PR. Factors promoting the tissue dissolving capability of sodium hypochlorite. Int Endod J 1982;15:187-96.

27. Van der Sluis WM, Gambarini G, 2, Wu MK, Wesselink PR. The influence of volume, type of irrigant and flushing method on removing artificially placed dentine debris from the apical root canal during passive ultrasonic irrigation. Int Endod J 2006;39:472-76. 